\title{
Single Dose Topical Application of Clotrimazole for the Treatment of Otomycosis: Is This Enough?
}

\author{
Rıza Dundar ${ }^{1}$ and İsmail İynen ${ }^{2}$ \\ ${ }^{1}$ Department of Otorhinolaryngology, Vocational School of Health Scienses, Acıbadem Mehmet Ali Aydınlar University, Eskişehir Hospital, \\ Eskişehir, Turkey \\ ${ }^{2}$ Department of Otorhinolaryngology, Harran University, Şanlıurfa, Turkey
}

\author{
Received June 13, 2018 \\ Revised July 31,2018 \\ Accepted August 14, 2018
}

Background and Objectives: To determine the efficacy of filling the external auditory meatus with $1 \%$ clotrimazole at a single visit for the treatment of otomycosis. Subjects and Methods: This prospective study included 40 patients who were referred to our clinic with complaints of ear itching, pain, and fullness, and were diagnosed with unilateral otomycosis. After cleaning the mycotic hyphae from the external auditory meatus, the ear canal was filled with $1 \%$ clotrimazole, using an intravenous catheter and syringe. The patients received follow-up examinations on post-treatment days 7, 15, and 45. Results: The follow-up otomicroscopic examinations revealed that $95 \%$ of the ear canals were entirely clean and that all symptoms had resolved. The post-treatment scores of pain, aural fullness and itching were significantly lower than the pre-treatment scores $(p<0.01)$. Conclusions: Filling the external auditory meatus with $1 \%$ clotrimazole at a single visit is an easy, efficient, and cost-effective treatment for otomycosis. Additionally, high patient compliance makes this treatment superior to long-term topical therapy.

J Audiol Otol 2019;23(1):15-19

\section{Introduction}

Otomycosis, a superficial fungal infection of the external auditory meatus, is generally seen in hot and moist climates worldwide and may present as acute, sub-acute, or chronic $[1,2]$. Factors that predispose patients to otomycosis are the use of wide-spectrum antibiotics, trauma to the external ear canal, and close contact with water [1-3]. Although a diverse group of microorganisms may be involved in the disease, $A s$ pergillus spp. and Candida spp. are the microorganisms most commonly isolated from the external ear canal of patients with otomycosis, with Penicillium spp., Mucor spp., and Rhizopus spp. seen less often [2-4]. Otomycosis usually presents as a superficial infection of the external ear canal skin. However, in cases with perforation of the tympanic membrane, the infection can also affect the middle ear. In rare cases, primari-

This is an Open Access article distributed under the terms of the Creative Commons Attribution Non-Commercial License (https://creativecommons.org/licenses/by-nc/4.0/) which permits unrestricted non-commercial use, distribution, and reproduction in any medium, provided the original work is properly cited. ly in immunocompromised individuals, the disease may be extensive and involve the temporal bone [5]. Otomycosis is frequently accompanied by bacterial infections.

Alkalinization of the ear canal and the consequent weakening of its cutaneous barrier produce conditions conducive to mycotic infections. Opportunistic fungal infections of inflamed and desquamated tissues give rise to the accumulation of debris containing hyphae in the external auditory meatus. Depending on the degree of infection, otomycosis may cause itching, pain, or fullness of the ear, as well as hearing loss, discharge, and tinnitus [2].

Multiple topical and oral antifungal agents are widely used in the treatment of otomycosis. Treatment usually requires use of antifungal drops for at least three weeks to prevent recurrence [6]. Eradication of the disease is hard and recurrences as high as $15 \%$ can be seen even with appropriate long term topical therapy [7]. Most of the time, patients' unwillingness in applying topical drops routinely for a long period of time, accounts for treatment failures. Therefore, we put in practice a "one-time only" application of an antifungal cream for the treat- 
ment of otomycosis and investigated the success of this single visit application. In the present study, we demonstrate that topical treatment applied by filling the external ear canal with $1 \%$ clotrimazole cream at a single visit is a cost-effective and efficient treatment modality for otomycosis.

\section{Subjects and Methods}

Patients who were referred to the ear, nose, and throat clinics at Harran University School of Medicine Hospital and diagnosed with unilateral otomycosis were enrolled in the study. This study was conducted following approval from the Ethics Committee of the Harran University School of Medicine (7405 $9997.50 .01 .04 / 30)$. The diagnosis of otomycosis was confirmed based on the history of symptoms and otomicroscopic observation of hyphae (Fig. 1). The informed consents of all of the patients were taken.

For each patient, the external ear canal and tympanic membrane were cleaned by aspiration under microscopic examination. Then, the external auditory meatus, beginning at the lateral surface of the tympanic membrane, was filled with $1 \%$ clotrimazole cream, using a green IV cannula and an insulin syringe (Fig. 2). The patients received follow-up examinations on days 7,15 , and 45 after cream administration. Wilcoxon test was used to compare the pre-treatment and post-treatment scores for statistical analysis.

\section{Results}

Forty patients diagnosed as unilateral otomycosis were enrolled in the study. The mean patient age was $28.6(21-65)$ years. There were 22 male and 18 female patients. Thirteen patients had central perforation of the tympanic membrane,

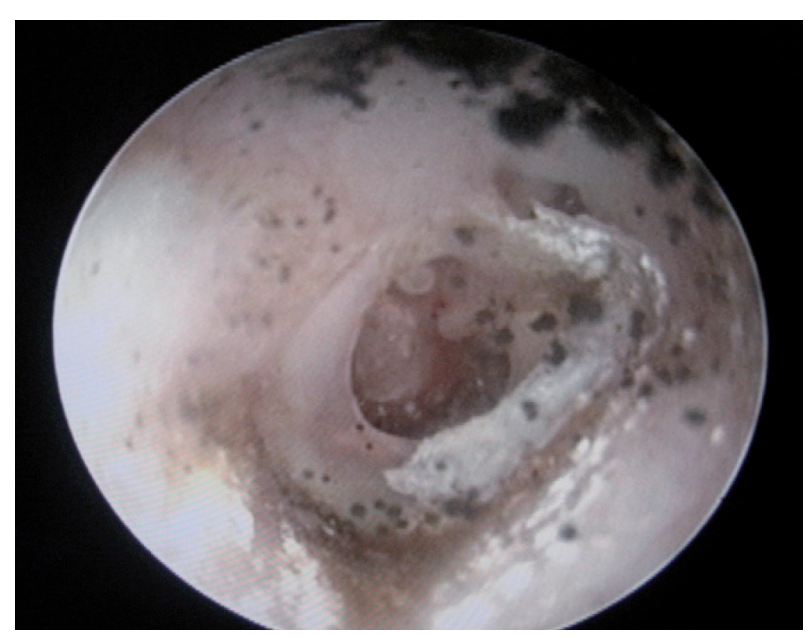

Fig. 1. Pretreatment otomicroscopic view of the external ear canal. two had a radical mastoidectomy cavity, and 25 patients had intact tympanic membranes and external ear canals. All of the patients reported itching of the ear, 14 patients had ear pain, 32 had symptoms of ear fullness and hearing loss, and eight patients presented with tinnitus.

After the treatment, 38 patients had recovered completely from the infection by the end of post-treatment day 7 . The remaining two patients denied any symptom regression. Otomicroscopic examinations of the patients who had recovered completely revealed that the external ear canal was totally clean, although some cream remained on parts of the tympanic membrane surface. Pain relief was seen at the end of day 2 . Pruritus began to decrease after 5-7 days and had totally resolved by days 10-12. Patients reported that tinnitus and fullness resolved after the external ear canal had been cleaned. Examinations on post-treatment day 15 showed complete resolution of symptoms, and microscopic examinations demonstrated complete eradication of infection and return of the ex-

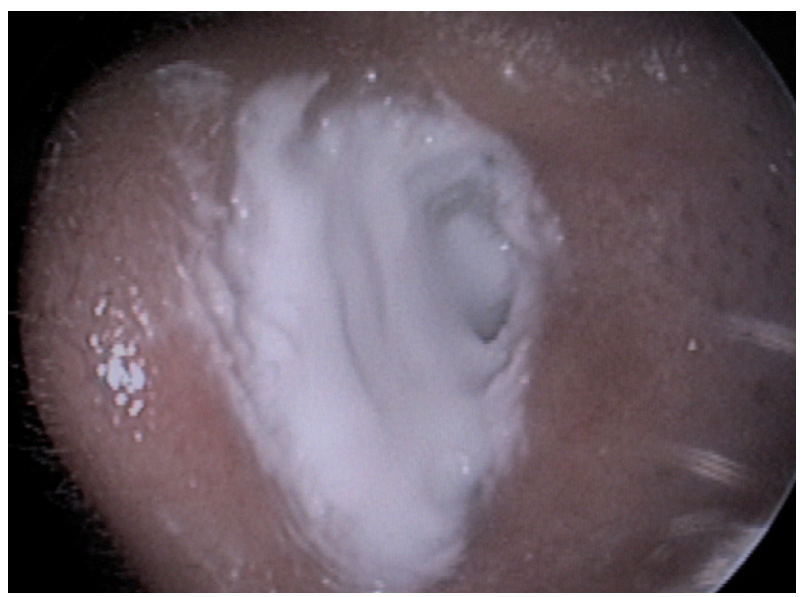

Fig. 2. The otomicroscopic view of the ear canal after filling with $1 \%$ clotrimazole cream.

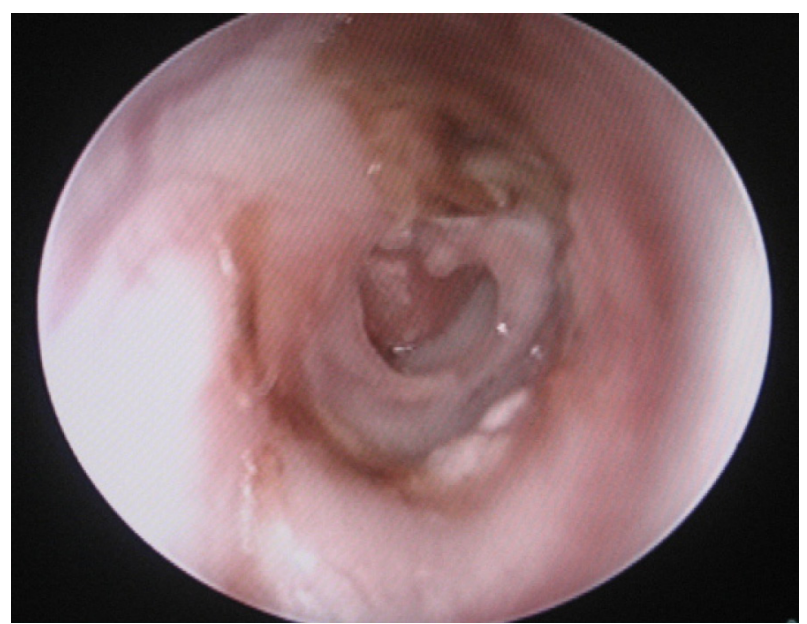

Fig. 3. The 15th day posttreatment otomicroscopic view of the external ear canal. 
ternal ear canal to its natural state (Fig. 3). The results of otomicroscopic examinations of the two patients who claimed no symptom relief were the same as the results for the other patients. These two patients complained mainly of a persistent itching sensation.

The distribution of pre-treatment and post-treatment scores of pain, aural fullness and itching of the cases were shown in Table 1. According to the statistical analysis, mean average post-treatment scores of the cases for pain, aural fullness and itching were significantly lower than the pre-treatment scores $(p<0.01)$ (Table 2, Fig. 4-6).

At 45 days after treatment, 39 of the 40 patients had no complaints. One patient experienced recurrence, which was treated again in the same manner as well as with an oral antifungal medication.

Table 1. The distribution of pre-treatment and post-treatment scores of pain, aural fullness and itching of the cases

\begin{tabular}{|c|c|c|c|c|}
\hline & \multicolumn{2}{|c|}{ Pre-treatment } & \multicolumn{2}{|c|}{ Post-treatment } \\
\hline & $n$ & $\%$ & $n$ & $\%$ \\
\hline \multicolumn{5}{|c|}{ Pain scores } \\
\hline 0 & 4 & 10.0 & 27 & 67.5 \\
\hline 1 & 10 & 25.0 & 10 & 25.0 \\
\hline 2 & 4 & 10.0 & 3 & 7.5 \\
\hline 3 & 7 & 17.5 & 0 & 0.0 \\
\hline 4 & 6 & 15.0 & 0 & 0.0 \\
\hline 5 & 9 & 22.5 & 0 & 0.0 \\
\hline \multicolumn{5}{|c|}{ Aural fullness } \\
\hline 0 & 2 & 5.0 & 17 & 42.5 \\
\hline 1 & 4 & 10.0 & 20 & 50.0 \\
\hline 2 & 2 & 5.0 & 3 & 7.5 \\
\hline 3 & 6 & 15.0 & 0 & 0.0 \\
\hline 4 & 15 & 37.5 & 0 & 0.0 \\
\hline 5 & 11 & 27.5 & 0 & 0.0 \\
\hline \multicolumn{5}{|l|}{ Itching } \\
\hline 0 & 0 & 0.0 & 17 & 42.5 \\
\hline 1 & 0 & 0.0 & 17 & 42.5 \\
\hline 2 & 0 & 0.0 & 6 & 15.0 \\
\hline 3 & 4 & 10.0 & 0 & 0.0 \\
\hline 4 & 10 & 25.0 & 0 & 0.0 \\
\hline 5 & 26 & 65.0 & 0 & 0.0 \\
\hline
\end{tabular}

\section{Discussion}

Otitis externa is a disease of the external ear canal seen in $5-20 \%$ of patients referred to ear, nose, and throat clinics. Although the disease is generally caused by bacteria, fungi may be the factor in $9-25 \%$ of cases. A fungal infection of the

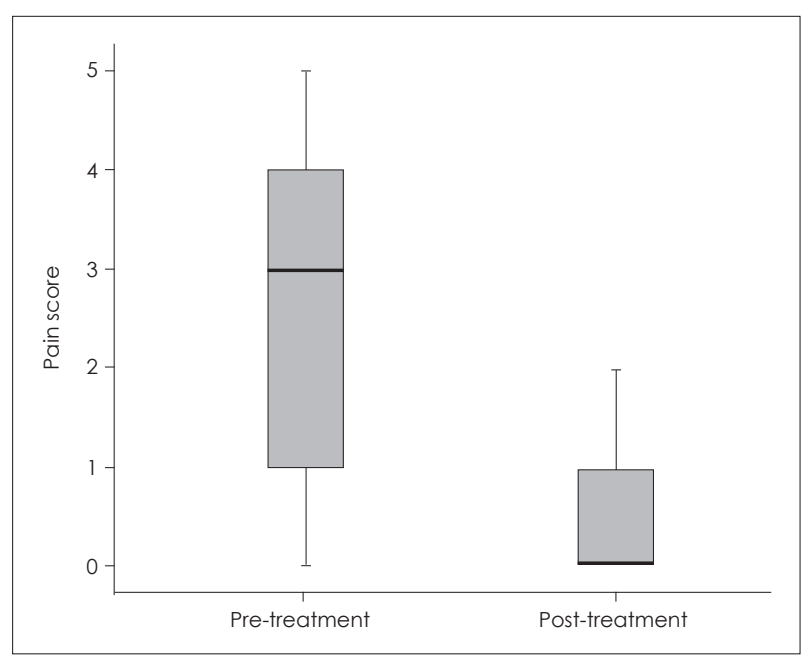

Fig. 4. The distribution of pre-treatment and post-treatment scores of pain.

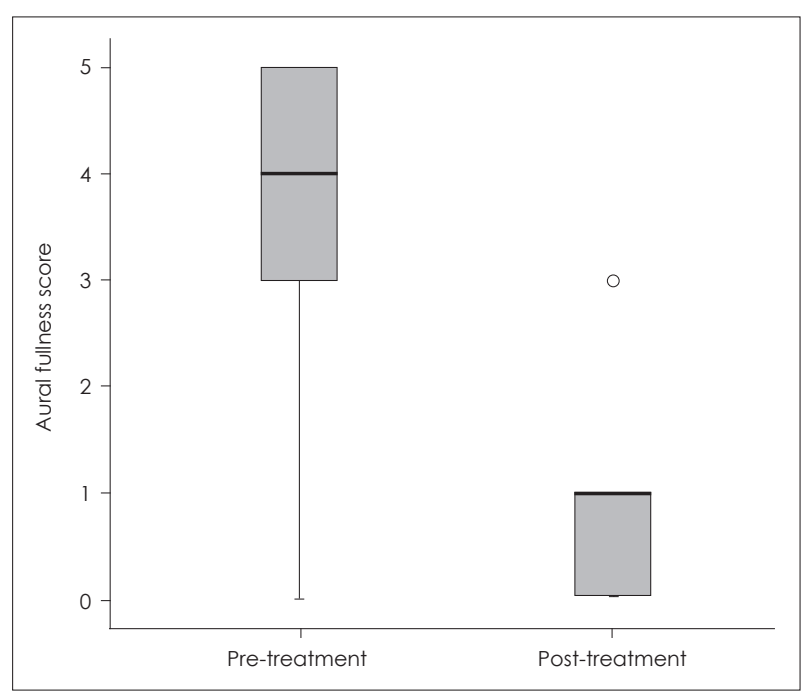

Fig. 5. The distribution of pre-treatment and post-treatment scores of aural fullness.

Table 2. Mean average of pre-treatment and post-treatment scores of pain, aural fullness and itching of the cases

\begin{tabular}{lccc}
\hline & Mean \pm SD & Median (min-max) & F* $^{*}$ \\
\hline Pain score (pre-treatment) & $2.7 \pm 1.7$ & $3(0-5)$ & $<0.001$ \\
Pain score (post-treatment) & $0.4 \pm 0.6$ & $0(0-2)$ & $<0.001$ \\
Aural fullness (pre-treatment) & $3.5 \pm 1.5$ & $4(0-5)$ & $<0.001$ \\
Aural fullness (post-treatment) & $0.7 \pm 0.8$ & $1(0-3)$ & $5(3-5)$ \\
Itching (pre-treatment) & $4.6 \pm 0.7$ & $1(0-2)$ & $<$ \\
Itching (post-treatment) & $0.7 \pm 0.7$ & & $<$
\end{tabular}

*Wilcoxon test 


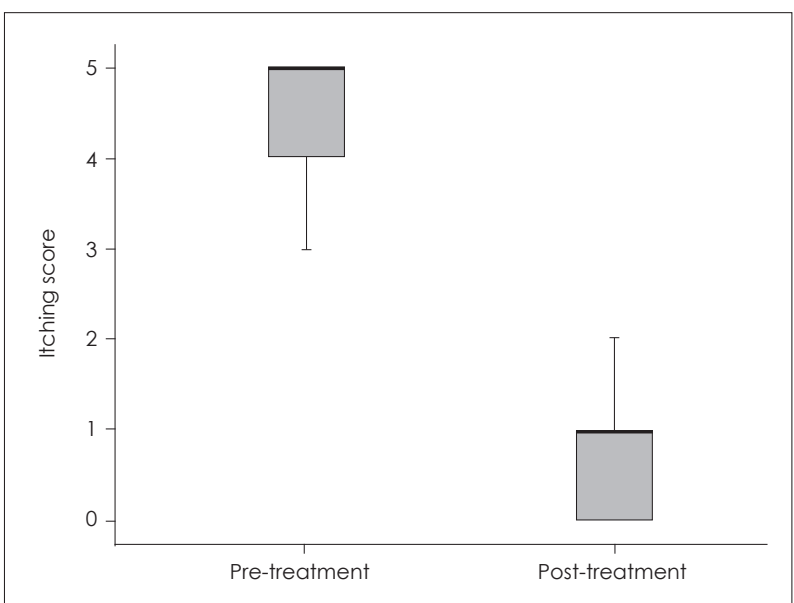

Fig. 6. The distribution of pre-treatment and post-treatment scores of Itching.

external ear canal is referred to as fungal otitis or otomycosis. In some cases, bacteria and fungi together may be the cause of infection. Fungal infections frequently occur along with the bacteria Staphylococcus aureus, Pseudomonas spp., and Proteus spp. Interestingly, the fungus Aspergillus fumigatus produces an antibiotic against $S$. aureus, so co-infection with these two organisms is not seen [1].

The fungi most commonly isolated from the external ear canal in otomycosis are Aspergillus spp. and Candida spp. Several studies have reported that Aspergillus spp. (A. niger, A. flavus, A. fumigatus) was found in the majority of cases, and Candida spp. (C. albicans, C. parapsilosis, C. tropicalis) were seen less frequently $[4,8,9]$.

Trichosporon spp. and Scedosporium spp. have also been isolated as causes of otomycosis. For example, Reiersöl [10] reported a case of otomycosis caused by Trichosporon cutaneum. Two species of Scedosporium, a filamentous fungus that lives in water, have been shown to cause otomycosis in humans; these are S. apiospermum and S. prolificans (S. inflatum). An important aspect of Scedosporium is its ability to cause severe and often fatal infections in immunocompromised patients [11,12].

Multiple predisposing factors play a role in inducing otomycosis. This infection is seen frequently in regions with tropical climates, where both high humidity and high temperatures occur. Inadequate personal hygiene, close contact of external ear canal with water, and attempts to clean the external ear canal with inappropriate objects also increase the risk for otomycosis, by altering the naturally acidic medium of the external ear canal or through traumatic maceration [13]. In addition, otomycosis is made more likely by the use of multiple and broad-spectrum antibiotics, steroids, or cytostatic medications, as well as the presence of neoplastic diseases or immunocompromised conditions.

Otomycosis affects primarily the squamous epithelium of the external ear canal, causing epithelial damage which results in symptoms of itching, aural fullness, and pain. As in the present study, itching and fullness are foreground symptoms. The external ear canal is edematous and oversensitive. White, black, and brown mycelium deposits can be seen in the external ear canal under otomicroscopic examination. A definitive diagnosis is possible with the culture of fungus in laboratory conditions $[4,14]$.

The first step in the treatment of otomycosis is the elimination of predisposing factors. Then, local cleaning and administration of an antifungal agent (local or systemic) are recommended $[7,13,14]$. Some studies report that giving ear drops, 3-4 times a day for 5-7 days, to increase the acidity of the ear canal is sufficient to treat the infection, while others suggest daily filling of the ear with an antifungal agent $[15,16]$.

Lucente [17] reported that thimerosal and m-cresyl acetate solutions are effective in vitro and that thimerosal is also effective against bacteria other than Enterococcus. However, thimerosal should be used for at least 14 days. Vennewald and Klemm [18] concluded that after local cleaning, treatment with a topical antifungal such as clotrimazole, miconazole, bifonazole, cyclopiroxolamine, and tolnaftate is sufficient for noninvasive fungal otitis externa, whereas oral antifungal agents such as itraconazole, voriconazole, and posaconazole, which give better penetration into bone and the nervous system, should be used to treat fungal malignant otitis externa, which can be complicated by meningitis and mastoiditis.

Munguia and Daniel [19] stated that the most effective agents for treating otomycosis are the azole antifungals, followed by nystatin and tolnaftate. In the present study, we administered topical clotrimazole to the patients diagnosed with otomycosis. Clotrimazole is an imidazole derivative with broad-spectrum antimycotic action. Clotrimazole inhibits the biosynthesis of ergosterol, a major component of the cell membranes of yeast and fungi. It replaces the precursor lanosterol as a substrate for the fungal cytochrome P450 enzyme lanosterol-14-demethylase, which catalyzes the conversion of lanosterol to ergosterol. This action alters the permeability of fungal cell walls [20]. In addition to their antimycotic properties, clotrimazole preparations appear to be safe and exhibit no evidence of ototoxicity in guinea pigs [21].

Mofatteh, et al. [22] conducted a study on 204 patients with otoycosis. In this study compared the recovery rates between topical betadine (povidoneiodine) and clotrimazole. Authors reported the efficacy of betadine and clotrimazole was the same for the treatment of otomycosis.

Romsaithong, et al. [23] conducted a study on 120 otomy- 
cosis patients were randomly assigned to receive either $1 \%$ clotrimazole solution (intervention group) or $3 \%$ boric acid in $70 \%$ alcohol (control group). This study reported $1 \%$ clotrimazole solution is more effective than $3 \%$ boric acid in $70 \%$ alcohol for otomycosis treatment.

In our study, we applied $1 \%$ clotrimazole cream locally to the external ear canal at a single visit. Such a treatment is more convenient than prescribing topical drops as it does not require patient's compliance or numerous visits for local cleaning. Another similar study is reported by van Hasselt, et al. [24] where they used a single instillation of silver nitrate gel for the treatment of otitis externa. They noted that this kind of a treatment saved both time and cost. Consequently, we believe that filling the external auditory meatus with $1 \%$ clotrimazole at a single visit after local cleaning, is a sufficient and cost-effective treatment modality. As antifungal treatments usually need to be administered 3-4 times a day, patient compliance is thought to be poor. With the treatment reported here, compliance would not be an issue.

\section{Conflicts of interest}

The authors have no financial conflicts of interest.

\section{REFERENCES}

1) Mugliston T, O'Donoghue G. Otomycosis--a continuing problem. J Laryngol Otol 1985;99:327-33.

2) Stern JC, Lucente FE. Otomycosis. Ear Nose Throat J 1988;67:8045, 809-10.

3) Zaror L, Fischman O, Suzuki FA, Felipe RG. Otomycosis in São Paulo. Rev Inst Med Trop Sao Paulo 1991;33:169-73.

4) Kaur R, Mittal N, Kakkar M, Aggarwal AK, Mathur MD. Otomycosis: a clinicomycologic study. Ear Nose Throat J 2000;79:606-9.

5) García-Martos P, García-Agudo R, Domínguez I, Noval JA. Otomicosis: aspectos clínicos y microbiológicos. Rev Diagn Biol 2001;50: $17-22$.

6) Abou-Halawa AS, Khan MA, Alrobaee AA, Alzolibani AA, Alshobaili HA. Otomycosis with perforated tympanic membrane: self medication with topical antifungal solution versus medicated ear wick. Int J Health Sci (Qassim) 2012;6:73-7.

7) Ho T, Vrabec JT, Yoo D, Coker NJ. Otomycosis: clinical features and treatment implications. Otolaryngol Head Neck Surg 2006;135:787-91.

8) Yehia MM, al-Habib HM, Shehab NM. Otomycosis: a common problem in north Iraq. J Laryngol Otol 1990;104:387-9.

9) Enweani IB, Igumbor H. Prevalence of otomycosis in malnourished children in Edo State, Nigeria. Mycopathologia 1997-1998;140:85-7.

10) Reiersol $S$. Trichosporon cutaneum isolated from a case of otomycosis. Acta Pathol Microbiol Scand 1955;37:459-63.

11) Milne LJ, McKerrow WS, Paterson WD, Petrie GR, Postlethwaite R. Pseudallescheriasis in northern Britain. J Med Vet Mycol 1986;24: 377-82.

12) Wilson CM, O'Rourke EJ, McGinnis MR, Salkin IF. Scedosporium inflatum: clinical spectrum of a newly recognized pathogen. J Infect Dis 1990;161:102-7.

13) Hurst WB. Outcome of 22 cases of perforated tympanic membrane caused by otomycosis. J Laryngol Otol 2001;115:879-80.

14) Jaiswal SK. Fungal infection of ear and its sensitivity pattern. Indian J Otolaryngol 1990;42:19-22.

15) Sander R. Otitis externa: a practical guide to treatment and prevention. Am Fam Physician 2001;63:927-36, 941-2.

16) Truitt TO, Tami TA. Otolaryngologic manifestations of human immunodeficiency virus infection. Med Clin North Am 1999;83:303-15.

17) Lucente FE. Fungal infections of the external ear. Otolaryngol Clin North Am 1993;26:995-1006.

18) Vennewald I, Klemm E. Otomycosis: diagnosis and treatment. Clin Dermatol 2010;28:202-11.

19) Munguia R, Daniel SJ. Ototopical antifungals and otomycosis: a review. Int J Pediatr Otorhinolaryngol 2008;72:453-9.

20) Smith EB. Topical antifungal drugs in the treatment of tinea pedis, tinea cruris, and tinea corporis. J Am Acad Dermatol 1993;28(5 Pt 1): S24-8.

21) Tom LW. Ototoxicity of common topical antimycotic preparations. Laryngoscope 2000;110:509-16.

22) Mofatteh MR, Naseripour Yazdi Z, Yousefi M, Namaei MH. Comparison of the recovery rate of otomycosis using betadine and clotrimazole topical treatment. Braz J Otorhinolaryngol 2018;84:404-9.

23) Romsaithong S, Tomanakan K, Tangsawad W, Thanaviratananich S. Effectiveness of 3 per cent boric acid in 70 per cent alcohol versus 1 per cent clotrimazole solution in otomycosis patients: a randomised, controlled trial. J Laryngol Otol 2016;130:811-5.

24) van Hasselt P, Gudde H. Randomized controlled trial on the treatment of otitis externa with one per cent silver nitrate gel. J Laryngol Otol 2004;118:93-6. 